\title{
Study on the barriers in magnetic tunnel junctions by electron holography
}

\author{
F. Shen, ${ }^{*}$ T. Zhu, ${ }^{* *}$ X. Xiang, ${ }^{* * *}$ John Q. Xiao*** and Z. Zhang ${ }^{1 *}$, \\ *Beijing Laboratory of Electron Microscopy, ${ }^{* *}$ State Key Laboratory for Magnetism, Institute of \\ Physics, Chinese Academy of Sciences, Beijing 100080, China \\ *** Department of Physics and Astronomy, University of Delaware, Newark, Delaware 19716
}

Recently, much attention has been focused on study of magnetic tunnel junctions (MTJs) for their fertile physics and potential applications ${ }^{[1]}$. The quality of the barrier and the oxidation degree of the ferromagnetic (FM) electrodes are critical for properties of MTJs. Consequently, efforts have been made to optimize plasma oxidation process and to prevent any side effects of oxidation on the underlying electrode ${ }^{[2]}$. However, there are little direct observations to reveal the energy profiles of oxide barriers in MTJs. In this report, we show some direct investigation of energy profiles of the barrier using off-axis electron holography $(\mathrm{EH})$ for the first time.

The studied MTJ structure is of $\mathrm{Si} / \mathrm{Ta}(5) / \mathrm{NiFe}(5) / \mathrm{FeMn}(12) / \mathrm{NiFe}(6) / \mathrm{AlO}_{\mathrm{x}} / \mathrm{NiFe}(6) / \mathrm{Ta}(5)$ with a wedge-shaped oxidized Al barrier layer. The numbers in the parentheses are the layer thickness in unit of $\mathrm{nm}$. As shown previously ${ }^{[3]}$ that the TMR ratio can only be reached to a maximum at certain thickness of Al layer in above MTJ structure, indicating an optimized oxidation condition of the Al layer. Fig. 1 shows a cross-sectional high-resolution electron microscopy (HREM) image of the optimum oxidized MTJ with a nominal Al thickness of about $1.26 \mathrm{~nm}^{[3]}$. From this image, we can see that the insulate oxide layer appears as a bright amorphous layer, which separates the top and bottom NiFe electrodes. It is interesting to note that the bottom electrode is with a strong (111) texture, while the top electrode has smaller grain sizes with random crystallographic orientations. This indicates that the grain boundary density is higher for the top electrode and the structural uniformity of the bottom is better. This type of structure nature holds for all of our MTJs samples with over- and under- oxidized barriers.

Owing to the fact that the energy profile of the barrier is the core issue for understanding the property of MTJs and it cannot be determined by the conventional HREM, an off-axis EH analysis was carried out in this study. Fig. 2 a) shows the off-axis electron hologram of a cross-sectional MTJ and Fig. 2b) is a corresponding phase image reconstructed by using Digital Micrograph software. The averaged phase profile (Fig.2 c)) can be obtained across the interfaces of $\mathrm{NiFe} / \mathrm{Al}_{2} \mathrm{O}_{3} / \mathrm{NiFe}$ in the phase image. It should be noted that here we interested in the interfaces of FMs/barrier, thus the contribution of magnetic field on the phase shift along the multi-layer normal can be neglected ${ }^{[4]}$. Therefore, the local phase shift can be expressed: $\Delta \Phi=C_{E} V_{0} t$, where $C_{E}$ is an interaction constant, $V_{0}$ the mean inner potential and $t$ the cross-sectional TEM sample thickness ${ }^{[4]}$. Since $t$ can be experimentally determined by using electron energy loss spectrum and it is a constant in our examined region, the phase shift in Fig.2 c) is mainly proportional to the barrier potential of the MTJ. From the energy profile, we can find out that the bottom interface is abrupt whereas the top

${ }^{1}$ Author to whom correspondence should be addressed; electronic mail address: zhang@blem.ac.cn 
interface has a visible slope. The abrupt profile indicated a sharp Oxide/FM interface, whereas the sloped one revealed a slight oxidation of the top electrode. This is in agreement with the HREM result as shown in Fig. 1, where the top FM electrode exhibits a 3D growth with small grains which introduced many grain boundaries connected to the oxide layer. Accordingly, the oxygen can readily diffuse into the top electrode through the grain boundaries, resulting an additional oxide layer from the top electrode. Above results suggest that a sharp top interface is difficult to obtain in the optimum oxidized MTJs, unless the growth quality of the top electrode on an amorphous $\mathrm{Al}_{2} \mathrm{O}_{3}$ layer can be significantly improved. The poorer top interface may be the main reason that the theoretical TMR values from Julière equation have never been achieved even in the “optimized” MTJ's.

In contrast to above HREM results where the structural features are all the same either for underor over-oxidized Al layers, the energy profiles look quite different for different oxidation conditions in our systematic studies. The profiles are more symmetrical at the top and bottom interfaces both for the under- and over-oxidized MTJs, but they are sharp for the former and sloped for the latter.

\section{References}

[1] J. S. Moodera et al., J. Magn. Magn. Mater. 200 (1999) 248; S. S. P. Parkin et al., J. Appl. Phys. 85 (1999) 5828; G. Prinz, Science 282 (1998) 1660, and references there in.

[2] W. Zhu etal., Appl. Phys. Lett. 78 (2001) 3103; J. Schmalhorst et al., J. Appl. Phys. 87 (2000) 5191; U. May et al., Appl. Phys. Lett. 78 (2001) 2026.

[3] T. Zhu et al., Phys. Rev. B 66 (2002) 094423; G. landry at al., Appl. Phys. Lett. 78 (2001) 501.

[4] J. C. H. Spence, in Experimental High Resolution Electron Microscopy (Oxford University Press, New York, 1988), 2nd ed; F. Shen et al., unpublished.

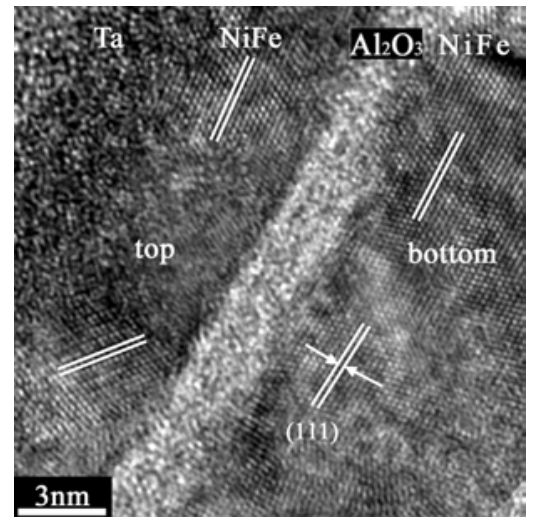

Fig1 The HREM image of the MTJ with optimum oxidized Al barrier. The pairs of white bars denote the $\{111\}$ plans of $\mathrm{NiFe}$.

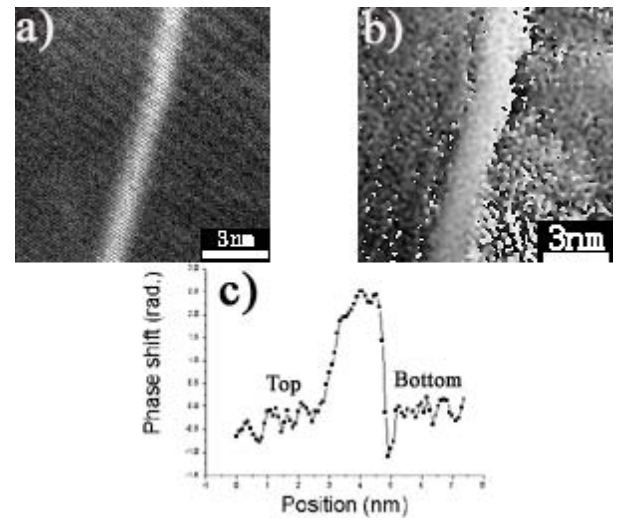

Fig.2 a)Hologram image of the MTJ junction with optimum oxidation; b)reconstructed phase image from a); c)averaged phase shift profiles. 